\title{
Synthesis, Molecular Docking Analysis, and Carbonic Anhydrase Inhibitory Evaluations of Benzenesulfonamide Derivatives Containing Thiazolidinone
}

\author{
Zuo-Peng Zhang, Ze-Fa Yin, Jia-Yue Li, Zhi-Peng Wang, Qian-Jie Wu, Jian Wang, Yang Liu *(-) and \\ Mao-Sheng Cheng * \\ Key Laboratory of Structure-Based Drug Design \& Discovery of Ministry of Education, \\ School of Pharmaceutical Engineering, Shenyang Pharmaceutical University, Shenyang 110016, China \\ * Correspondence: y.liu@syphu.edu.cn (Y.L.); mscheng@syphu.edu.cn (M.-S.C.)
}

Received: 31 May 2019; Accepted: 27 June 2019; Published: 30 June 2019

\begin{abstract}
To find novel human carbonic anhydrase ( $h \mathrm{CA}$ ) inhibitors, we synthesized thirteen compounds by combining thiazolidinone with benzenesulfonamide. The result of the X-ray single-crystal diffraction experiment confirmed the configuration of this class of compounds. The enzyme inhibition assays against $h$ CA II and IX showed desirable potency profiles, as effective as the positive controls. The docking studies revealed that compounds (2) and (7) efficiently bound in the active site cavity of $h$ CA IX by forming sufficient interactions with active site residues. The fragment of thiazolidinone played an important role in the binding of the molecules to the active site.
\end{abstract}

Keywords: human carbonic anhydrase IX; thiazolidinone; benzenesulfonamide; docking study

\section{Introduction}

Human carbonic anhydrases ( $h \mathrm{CAs}$, EC 4.2.1.1) are important metalloenzymes that can catalyze the interconversion of $\mathrm{CO}_{2}$ and $\mathrm{H}_{2} \mathrm{O}$ with $\mathrm{HCO}_{3}{ }^{-}$and $\mathrm{H}^{+}$. These enzymes play a pivotal role in many cellular and physiological processes, such as electrolyte secretion, $\mathrm{pH}$ homeostasis, respiration, and biosynthetic pathways. There are at least $15 \mathrm{CA}$ isoforms in humans, which differ in molecular properties, subcellular localization, and tissue distribution [1-4]. Uniquely, CA IX has low expression in many normal tissues, but it is overexpressed in numerous solid tumors, assisting in tumor cell survival, proliferation, and metastasis in the tumor microenvironment [5-11]. Therefore, CA IX has been validated as a promising target for cancer diagnostics and treatments. Currently, there are a large number of CA IX inhibitors reported in the literature, but only SLC-0111 (Figure 1) has completed Phase I clinical trials [12-14].<smiles>NS(=O)(=O)c1ccc(NC(=O)Nc2ccc(F)cc2)cc1</smiles>

Figure 1. The chemical structure of SLC-0111.

In the area of medicinal chemistry, five-membered heterocyclic compounds, especially heterocycles having more than one hetero atom, have shown a diverse range of biological activities. Among them, thiazolidinone, which bears a sulfur atom at 1-position, a nitrogen atom at 3-position and a carbonyl group at 2-, 4-, or 5-positions, has received a great deal of attention over the years since it is a significant and versatile moiety that has occupied a prominent position in medicinal chemistry [15-24]. Derivatives 
containing a thiazolidinone moiety have demonstrated a wide range of pharmacological properties, including anti-HIV, anti-malarial, anti-inflammatory and hypoglycaemic activities [25-27]. In many cases, the introduction of thiazolidinone into molecules can enhance the interaction between the whole molecule and the biological target. In the field of anti-tumor drugs, thiazolidinone fragments are also frequently chosen to modify lead compounds. A variety of thiazolidinone-based derivatives with different substituents are being considered as potential anticancer agents [24,28]. In particular, previous work by the Azam group pointed out that introducing a five-membered heterocyclic ring in some compounds can help increase $h$ CA IX inhibitory activity, achieving Ki values in the low micromolar range [28]. Supuran and Akdemir's work also drew the same conclusion [29].

To extend the applicability of thiazolidinone to CA inhibitors, in this work, we synthesized thirteen novel compounds by combining sulfonamide with thiazolidinone. These compounds were then tested for enzyme inhibition of $h$ CA IX and off-target $h$ CA II isoform (as a subtype of the CA family, CA II is widely distributed in the body and participates in various physiological activities). The inhibitory activities were determined to be in the low nanomolar region. To determine the binding mechanism and rationalize the trend of the inhibition profiles, molecular docking studies of the designed hybrids in the active site of $h$ CA IX and II were carried out, respectively.

\section{Results and Discussion}

\subsection{Chemistry}

The general synthetic route for the synthesis of the target benzenesulfonamide-thiazolidinone derivatives is shown in Scheme 1. Isothiocyanatobenzene reacted with mafenide hydrochloride to afford benzenesulfonamide (1) as the intermediate, which continued in the next cyclization reaction to form compound (2). Thereafter, compounds (3-14) were obtained via the Knoevenagel condensation of compound (2) reacting with different substituted benzaldehydes.

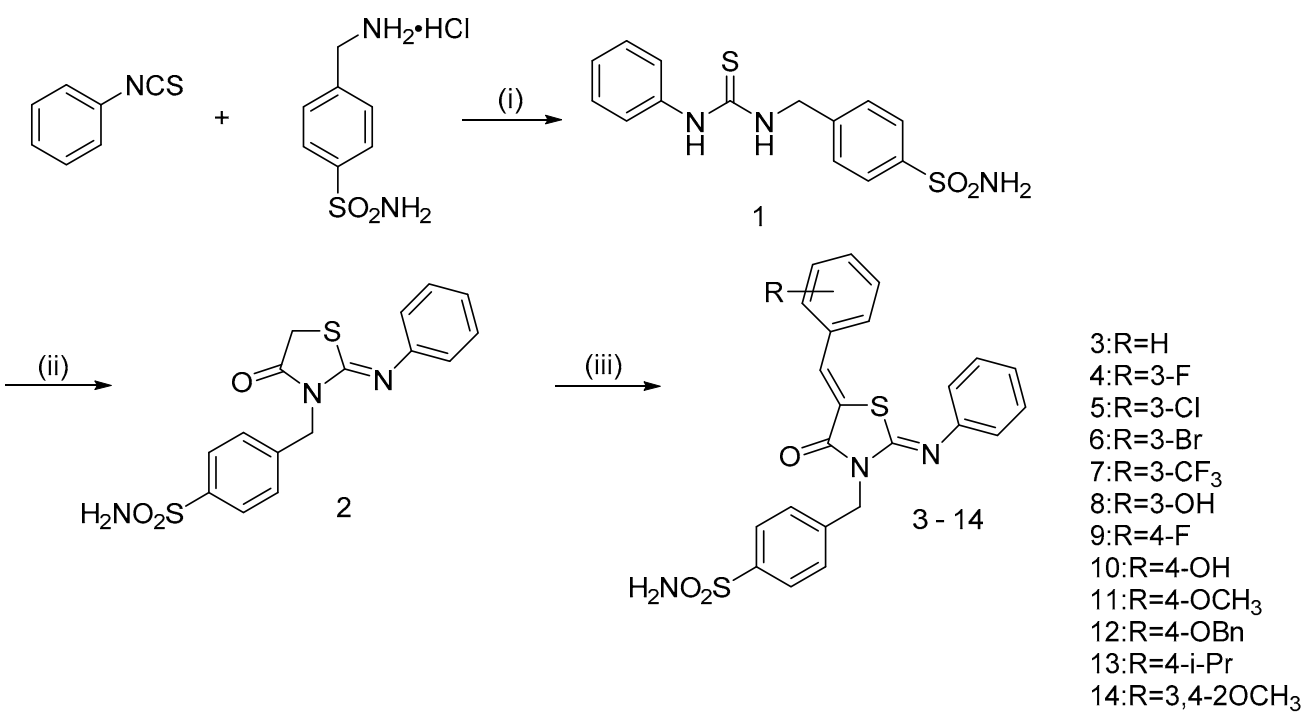

Scheme 1. The general synthesis route of compounds 3-14. Reagents and conditions: (i) $\mathrm{NaHCO}_{3}$, DMF 4 Å MS, r.t., 4 h (ii) chloroacetic acid, NaOAc, EtOH, reflux, 12 h (iii) substituted benzaldehydes, piperidine, $\mathrm{EtOH}$, reflux, $5 \mathrm{~h}$.

\section{2. hCA Enzyme Inhibition Studies}

The synthesized compounds (2-14) were evaluated for inhibitory activity against $h$ CA II and IX and by monitoring the hydrolysis of 4-nitrophenyl acetate (4-NPA). The inhibition data are summarized in Table 1. 
Table 1. Inhibitory activity of compounds (2-14) against $h$ CAs and molecular docking binding scores.

\begin{tabular}{|c|c|c|c|c|}
\hline Compound & $\mathbf{R}$ & $h \mathrm{CA}$ II $\left(\mathrm{IC}_{50}, \mathrm{nM}\right)$ & $h$ CA IX $\left(\mathrm{IC}_{50}, \mathrm{nM}\right)$ & $\begin{array}{l}\text { Docking Scores } \\
\text { (Kcal/mol) }\end{array}$ \\
\hline 2 & / & 266.2 & 242.1 & -5.16 \\
\hline 3 & $\mathrm{H}$ & 166.3 & 182.9 & -6.14 \\
\hline 4 & $3-\mathrm{F}$ & 146.5 & 130.5 & -6.68 \\
\hline 5 & $3-\mathrm{Cl}$ & 270.3 & 292.3 & -5.00 \\
\hline 6 & $3-\mathrm{Br}$ & 148.0 & 154.2 & -6.18 \\
\hline 7 & $3-\mathrm{CF}_{3}$ & 335.0 & 544.4 & -3.24 \\
\hline 8 & $3-\mathrm{OH}$ & 151.3 & 140.9 & -6.90 \\
\hline 9 & $4-\mathrm{F}$ & 216.7 & 246.1 & -5.02 \\
\hline 10 & $4-\mathrm{OH}$ & 160.8 & 144.9 & -6.30 \\
\hline 11 & $4-\mathrm{OCH}_{3}$ & 115.4 & 117.2 & -6.79 \\
\hline 12 & $4-\mathrm{OBn}$ & 1725 & $>10,000$ & 0.70 \\
\hline 13 & 4-i-Pr & 692.1 & 1246 & -2.15 \\
\hline 14 & $3,4-2 \mathrm{OCH}_{3}$ & 121.3 & 146.5 & -6.51 \\
\hline Mafenide hydrochloride & & 8912 & $>30,000$ & $\mathrm{NA}^{\mathrm{a}}$ \\
\hline AcetazolaMide (AZM) & & 30.47 & 88.1 & -5.46 \\
\hline SLC-0111 & & 137.8 & 180.7 & -6.01 \\
\hline
\end{tabular}

a Not available. Autodock is not suitable to score mafenide hydrochloride as a salt-type compound. The value of mafenide's docking score is -5.06 .

According to the data in Table 1, the structure-activity relationships (SAR) of compounds (2-14) are preliminarily summarized below.

i. Compared to the lead compound mafenide hydrochloride, most synthesized compounds showed a significant increase in the inhibitory activity against both $h$ CA II and IX, which were comparable to the positive control drugs AZM and SLC-0111, except for the compound (12) and (13). There was a certain relationship between the molecular docking binding scores with the value of $\mathrm{IC}_{50}$, and the compound with better in vitro activity owned more negative score value.

ii. Compounds (3-14) were synthesized by introducing substituted aromatic groups into the adjacent carbonyl group of thiazolidinone. In most cases, the introduction of an electron-withdrawing group into the benzenesulfonamide-thiazolidinone scaffold caused a clear decrease in the inhibitory potencies against $h$ CA. Electron-donating groups provided better inhibition results, but when there was more than one electron-donating group, the activity of the compound decreased slightly as in compound (14).

iii. Among the halogenated benzene substituted derivatives (4-6) with the halogen at the meta position, the fluorine substituted derivative showed the highest inhibitory activity. Comparing compounds (4) and (8)-(10), we found that the compounds with substituents in the meta position showed better inhibition activity than the ones with substituents in the para position.

iv. The weakest compound among the series was the benzyloxy substituted compound (12). The isopropyl substituted compound (13) also exhibited poor inhibitory activity. Therefore, we can conclude that increased steric hindrance of the substituents decreases the inhibition of $h \mathrm{CA}$. However, the effect of larger steric hindrance on the inhibitory activity of $h$ CA II was not as great as that of $h$ CA IX. 


\subsection{Docking Studies into the Active Site of $h \mathrm{CAs}$}

To further annotate the binding mode of the series compounds, compounds (4), (7), (11) and 5FL4-ref were chosen for docking studies into the active site of $h$ CA IX. The results were shown in Figure 2.

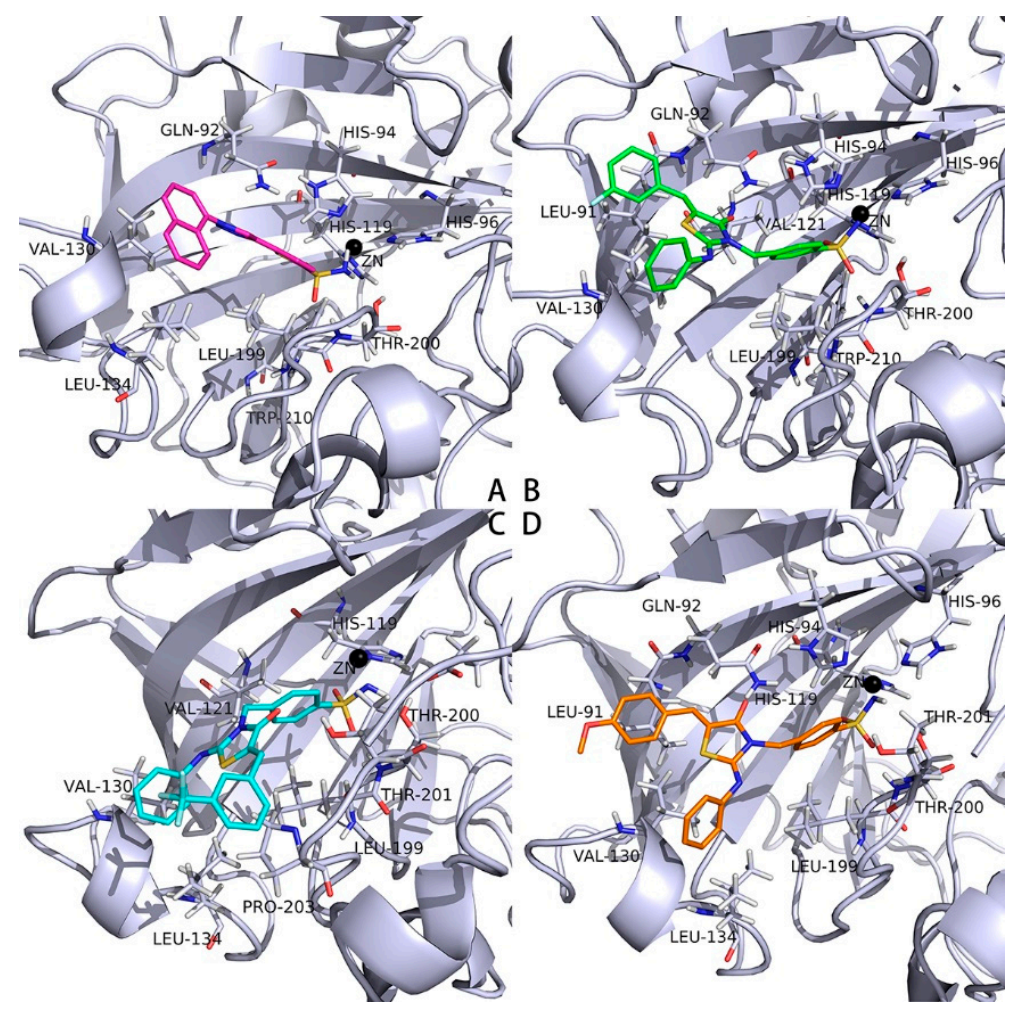

Figure 2. The study of binding mode within CA IX. (A) 5FL4-ref (pink) (B) compound (4) (green) (C) compound (7) (cyan) (D) compound (11) (khaki).

As shown in part A of Figure 2, compound 5FL4-ref interacted with the active site of CA IX by one hydrogen bond between the Thr200 and the $\mathrm{SO}_{2}$ group. Additionally, the nitrogen atom of the sulfonamide group formed a coordination bond with the zinc ion. The naphthyl moiety of 5FL4-ref formed interactions with Val130 and Leu134, and the heterocycle moiety of 5FL4-ref interacted with Gln92 and Thr199 via hydrogen and hydrophobic bond, respectively. The residues with which compounds (4), (7) and (11) interacted were essentially identical. Compounds (4), (7) and (11) also formed interactions with the zinc cation by coordinating the nitrogen atom of the sulfonamide. However, the interaction with the important residue Val130 was formed by the fragment of thiazolidinone in compounds (4), (7) and (11) (part B, C and D of Figure 2). At the same time, carbonyl groups in the thiazolidinone fragments of compounds (4) and (11) also formed hydrogen bonds with Gln92. Due to the rigidity of the heterocycle fragments in molecules, the substituted phenyl groups of compounds (4) and (11) achieved the same torsion angle in the active site when bound to Gln92. Due to the presence of trifluoromethyl, the conformation of compound (7) was slightly different from that of other two compounds and did not form interaction with residues Leu91 and Gln92, which may be the reason for the weaker activity of compound (7) than that of compounds (4) and (11). In the enzyme inhibition tests, the series of compounds also showed quite good inhibitory activity against $h$ CA II. To illustrate the reasons, compounds (4), (7) and (11) and 4IWZ-ref were chosen for docking studies into the active site of $h$ CA II. The results were shown in Figure 3. 


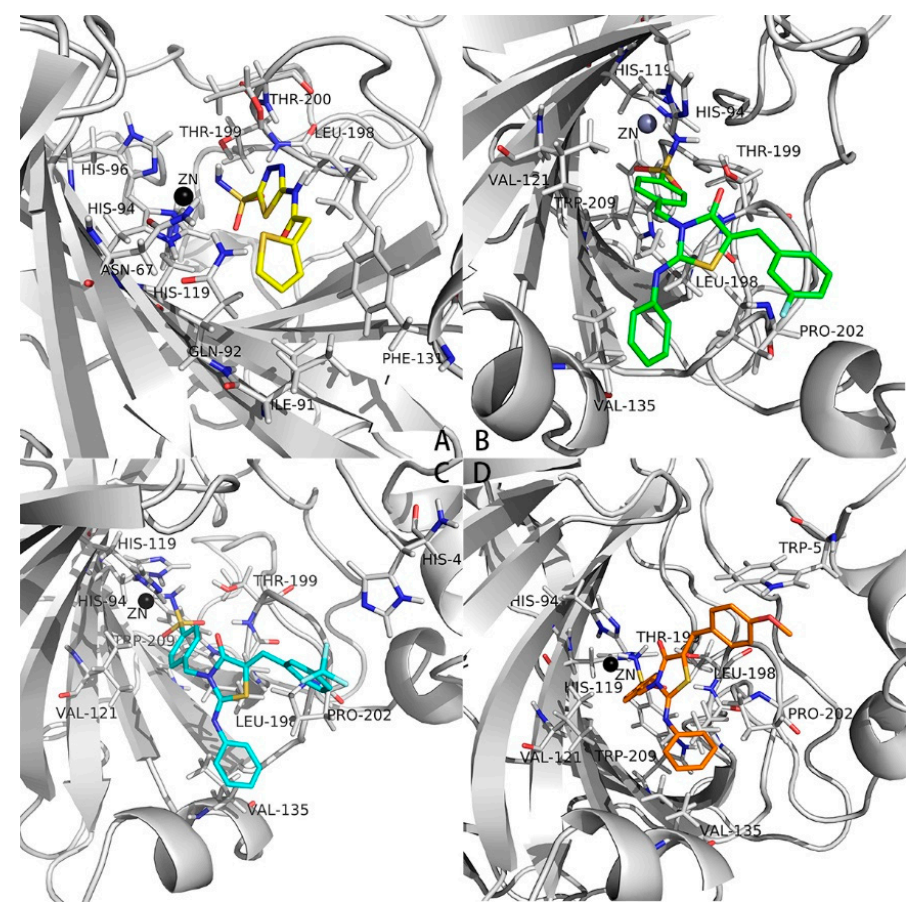

Figure 3. The study of binding mode within CA II. (A) 4IWZ-ref (yellow) (B) compound (4) (green) (C) compound (7) (cyan) (D) compound (11) (khaki). As shown in part A of Figure 3, compound 4IWZ-ref interacted with the active site of CA II by one hydrogen bond between the Thr199 and the $\mathrm{SO}_{2}$ group. Additionally, the nitrogen atom of the sulfonamide group formed a coordination bond with the zinc ion. The thiadiazole fragment of 4IWZ-ref formed interactions with Leu198, and the thiophene fragment of 4IWZ-ref interacted with some residues including Asn67, Ile91, Gln92 and Phe131. As shown in part B, $\mathrm{C}$ and D of Figure 3, the residues interacting with compounds (4), (7) and (11) at the active site were substantially identical. The different conformation and torsion angle of compounds in the active cavity led to the difference of activity.

Although most of the compounds showed good inhibitory activity, some of them showed little activity. To explain this phenomenon, the dominant conformations of compounds (2), (3), (12) and (13) in CA IX were superimposed. As shown in Figure 4, compounds (2) and (3) with better activity maintained almost the same conformation in the active site, whereas compounds (12) and (13) underwent considerable torsion, which led to an increase in the distance between the nitrogen atom of the sulfonamide group and the zinc ion. The increased distance may be the reason for the poor activities of these two compounds.

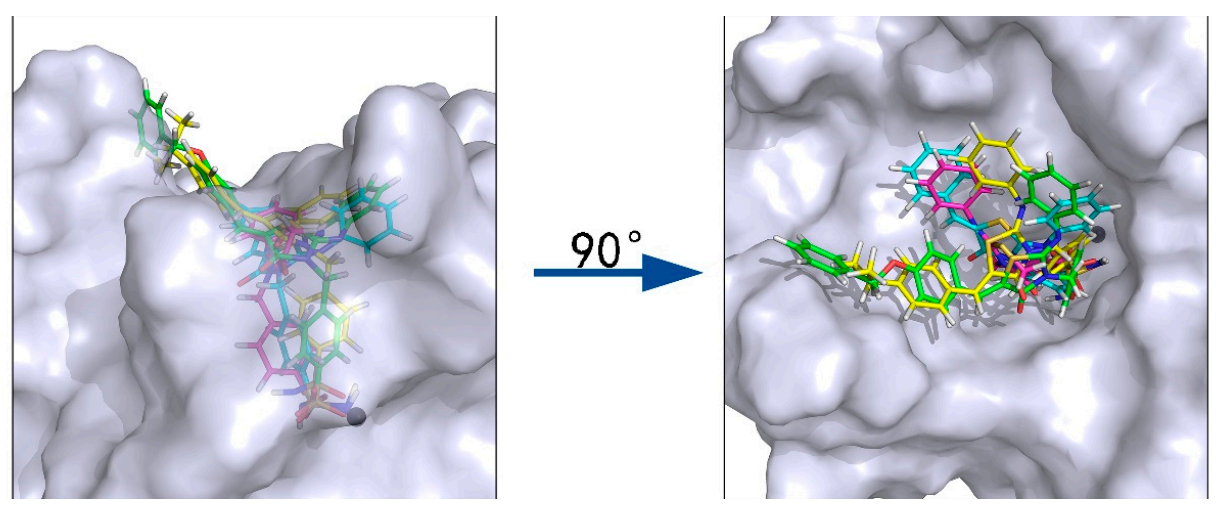

Figure 4. Compounds superposed in CA IX active site: compound (2) (pink), compound (3) (turquoise), compound (12) (green), compound (13) (yellow). 


\section{Materials and Methods}

\subsection{Chemistry}

All the reagents (Energy Chemical, Shanghai, China) were used without further purification unless otherwise specified. Solvents were dried and redistilled prior to use in the usual manner. Analytical TLC was per-formed using silica gel $\mathrm{HF}_{254}$ (Qingdao Haiyang Chemical, Qingdao, China). Preparative column chromatography was performed with silica gel $\mathrm{H}$. Melting points were obtained on a Büchi melting point B-540 apparatus. ${ }^{1} \mathrm{H}$ and ${ }^{13} \mathrm{C}$ NMR spectra (details of raw data for compounds, see Figures S1-S26) were recorded on a Bruker ARX $600 \mathrm{MHz}$ or $400 \mathrm{MHz}$ spectrometer (Bruker, Zurich, Switzerland) HRMS were obtained on an Agilent ESI-QTOF instrument (Agilent, Santa Clara, CA, USA).

\subsubsection{4-((3-phenylthioureido)methyl)benzenesulfonamide (1)}

Compound (1) was prepared according to the literature procedures [28]. To a solution of mafenide hydrochloride $(1.0 \mathrm{~g}, 4.5 \mathrm{mmol})$ and isothiocyanatobenzene $(0.54 \mathrm{~mL}, 4.5 \mathrm{mmol})$ in DMF $(10 \mathrm{~mL})$ sodium bicarbonate $(0.42 \mathrm{~g}, 5.0 \mathrm{mmol})$ and molecular sieves $(4 \AA, 1.0 \mathrm{~g})$ were added. The mixture was stirred under room temperature for $4 \mathrm{~h}$. After the molecular sieves were filtered off, $15 \mathrm{~mL}$ of water was added. The mixture was stirred vigorously to form white solids, which were filtered off to afford compound (1) $(0.99 \mathrm{~g}, 68 \%)$.

\subsection{2. (Z)-4-((4-oxo-2-(phenylimino)thiazolidin-3-yl)methyl)benzenesulfonamide (2)}

To a mixture of compound $(\mathbf{1})(0.55 \mathrm{~g}, 1.7 \mathrm{mmol})$ and chloroacetic acid $(0.24 \mathrm{~g}, 2.6 \mathrm{mmol})$ in EtOH $(20 \mathrm{~mL})$, sodium acetate anhydrous $(0.28 \mathrm{~g}, 3.4 \mathrm{mmol})$ was added. The mixture was stirred at $78{ }^{\circ} \mathrm{C}$ for $12 \mathrm{~h}$. The solvent was removed under reduced pressure, and the resulting residue was dissolved in ethyl acetate and washed by water. Then, ethyl acetate was removed under reduced pressure, affording light yellow solid, which was recrystallized from ethanol to give compound (2) as white powder $(0.28 \mathrm{~g}$, 45\%). m.p. $179.2-180.3{ }^{\circ} \mathrm{C} ;{ }^{1} \mathrm{H}-\mathrm{NMR}\left(\mathrm{DMSO}-\mathrm{d}_{6}, 600 \mathrm{MHz}\right) \delta 7.81(\mathrm{~d}, J=8.1 \mathrm{~Hz}, 2 \mathrm{H}), 7.55(\mathrm{~d}, J=8.2 \mathrm{~Hz}$, $2 \mathrm{H}), 7.42-7.29(\mathrm{~m}, 4 \mathrm{H}), 7.13(\mathrm{t}, J=7.4 \mathrm{~Hz}, 1 \mathrm{H}), 6.90(\mathrm{~d}, J=7.4 \mathrm{~Hz}, 2 \mathrm{H}), 4.99(\mathrm{~s}, 2 \mathrm{H}), 4.13(\mathrm{~s}, 2 \mathrm{H}) ;{ }^{13} \mathrm{C}$ NMR (DMSO-d $\left.d_{6}, 151 \mathrm{MHz}\right) \delta 172.49,155.47,148.28,143.66,140.48,129.79,128.49,126.32,124.88,121.33$, 45.45, 33.10; HRMS (ESI): Calcd. for $[\mathrm{M}+\mathrm{Na}]^{+} \mathrm{C}_{16} \mathrm{H}_{15} \mathrm{~N}_{3} \mathrm{O}_{3} \mathrm{~S}_{2}$ : 361.0555, Found 361.0548.

3.1.3. General Procedure of Synthesis of 4-((Z)-5-((Z)-benzylidene)-4-oxo-2-(phenylimino)thiazolidin3-yl)methyl)benzenesulfonamide hybrids (3-14)

A mixture of compound (2) $(0.14 \mathrm{~g}, 0.39 \mathrm{mmol})$, different aldehydes $(0.39 \mathrm{mmol})$, piperidine $(0.43 \mathrm{mmol}, 0.040 \mathrm{~mL})$, and ethanol $(25 \mathrm{~mL})$ were refluxed for $5 \mathrm{~h}$. The reaction mixture was cooled to room temperature. The solvent was removed under reduced pressure, and the resulting residue was purified by column chromatography (150:1, dichloromethane-methanol) to give compounds (3-14), respectively.

4-(((Z)-5-((Z)-benzylidene)-4-oxo-2-(phenylimino)thiazolidin-3-yl)methyl)benzenesulfonamide (3): Light yellow powder, m.p. 227.0-229.3 ${ }^{\circ} \mathrm{C}, 42 \%$ yield; ${ }^{1} \mathrm{H}-\mathrm{NMR}\left(\mathrm{CD}_{3} \mathrm{CN}, 600 \mathrm{MHz}\right) \delta 7.89(\mathrm{~d}, \mathrm{~J}=8.4 \mathrm{~Hz}$, 2H), $7.79(\mathrm{~s}, 1 \mathrm{H}), 7.66(\mathrm{~d}, J=8.4 \mathrm{~Hz}, 2 \mathrm{H}), 7.53(\mathrm{~d}, J=7.4 \mathrm{~Hz}, 2 \mathrm{H}), 7.48(\mathrm{dd}, J=10.2,4.8 \mathrm{~Hz}, 2 \mathrm{H}), 7.43$ $(\mathrm{t}, J=7.9 \mathrm{~Hz}, 3 \mathrm{H}), 7.24(\mathrm{t}, J=7.5 \mathrm{~Hz}, 1 \mathrm{H}), 7.05-7.00(\mathrm{~m}, 2 \mathrm{H}), 5.68(\mathrm{~s}, 2 \mathrm{H}), 5.22(\mathrm{~s}, 2 \mathrm{H}) ;{ }^{13} \mathrm{C}-\mathrm{NMR}\left(\mathrm{CD}_{3} \mathrm{CN}\right.$, $151 \mathrm{MHz}) \delta 166.87,150.88,148.55,143.13,141.45,134.21,131.29,130.60,130.42,130.07,129.74,129.17$, 126.86, 125.59, 122.15, 121.60, 46.16; HRMS (ESI): Calcd. for $[\mathrm{M}+\mathrm{Na}]^{+} \mathrm{C}_{23} \mathrm{H}_{19} \mathrm{~N}_{3} \mathrm{O}_{3} \mathrm{~S}_{2}: 449.0868$, Found 449.0872 .

4-(((Z)-5-((Z)-3-fluorobenzylidene)-4-oxo-2-(phenylimino)thiazolidin-3-yl)methyl)benzenesulfonamide (4): Light yellow powder, m.p. $226.9-227.6{ }^{\circ} \mathrm{C}, 45 \%$ yield; ${ }^{1} \mathrm{H}-\mathrm{NMR}\left(\mathrm{CD}_{3} \mathrm{CN}, 600 \mathrm{MHz}\right) \delta 7.89$ $(\mathrm{d}, J=8.4 \mathrm{~Hz}, 2 \mathrm{H}), 7.72(\mathrm{~s}, 1 \mathrm{H}), 7.70-7.64(\mathrm{~m}, 3 \mathrm{H}), 7.59(\mathrm{dd}, J=8.0,0.9 \mathrm{~Hz}, 1 \mathrm{H}), 7.50(\mathrm{~d}, J=7.9 \mathrm{~Hz}, 1 \mathrm{H})$, $7.44(\mathrm{t}, J=7.9 \mathrm{~Hz}, 2 \mathrm{H}), 7.40(\mathrm{t}, J=7.9 \mathrm{~Hz}, 1 \mathrm{H}), 7.25(\mathrm{t}, J=7.4 \mathrm{~Hz}, 1 \mathrm{H}), 7.03(\mathrm{~d}, J=7.4 \mathrm{~Hz}, 2 \mathrm{H}), 5.68$ 
(s, 2H), $5.22(\mathrm{~s}, 2 \mathrm{H}) ;{ }^{13} \mathrm{C}-\mathrm{NMR}\left(\mathrm{CD}_{3} \mathrm{CN}, 151 \mathrm{MHz}\right) \delta 166.76,150.52,148.57,143.33,141.46,136.65,133.33$, 131.68, 130.26, 129.62, 129.39, 128.72, 127.01, 125.85, 124.11, 123.26, 121.70, 46.43; HRMS (ESI): Calcd. for $[\mathrm{M}+\mathrm{Na}]^{+} \mathrm{C}_{23} \mathrm{H}_{18} \mathrm{FN}_{3} \mathrm{O}_{3} \mathrm{~S}_{2}: 467.0774$, Found 467.0779.

4-(((Z)-5-((Z)-3-chlorobenzylidene)-4-oxo-2-(phenylimino)thiazolidin-3-yl)methyl)benzenesulfonamide (5): Light yellow powder, m.p. $218.9-220.9{ }^{\circ} \mathrm{C}, 41 \%$ yield; ${ }^{1} \mathrm{H}-\mathrm{NMR}\left(\mathrm{CD}_{3} \mathrm{CN}, 600 \mathrm{MHz}\right) \delta 7.89(\mathrm{~d}, J=8.4 \mathrm{~Hz}$, $2 \mathrm{H}), 7.72(\mathrm{~s}, 1 \mathrm{H}), 7.66(\mathrm{~d}, J=8.4 \mathrm{~Hz}, 2 \mathrm{H}), 7.52(\mathrm{~s}, 1 \mathrm{H}), 7.47-7.41(\mathrm{~m}, 5 \mathrm{H}), 7.25(\mathrm{t}, J=7.5 \mathrm{~Hz}, 1 \mathrm{H}), 7.02$ $(\mathrm{dd}, J=8.3,0.9 \mathrm{~Hz}, 2 \mathrm{H}), 5.68(\mathrm{~s}, 2 \mathrm{H}), 5.22(\mathrm{~s}, 2 \mathrm{H}) ;{ }^{13} \mathrm{C}-\mathrm{NMR}\left(\mathrm{CD}_{3} \mathrm{CN}, 151 \mathrm{MHz}\right) \delta 165.23,149.00,147.03$, 141.78, 139.92, 134.86, 133.65, 129.94, 128.83, 128.81, 128.15, 127.84, 126.84, 125.47, 124.30, 122.59, 120.15, 44.89; HRMS (ESI): Calcd. for [M + Na] ${ }^{+} \mathrm{C}_{23} \mathrm{H}_{18} \mathrm{ClN}_{3} \mathrm{O}_{3} \mathrm{~S}_{2}: 483.0478$, Found 483.0492 .

4-(((Z)-5-((Z)-3-bromobenzylidene)-4-oxo-2-(phenylimino)thiazolidin-3-yl)methyl)benzenesulfonamide (6): Light yellow powder, m.p. $217.7-220.3{ }^{\circ} \mathrm{C}, 48 \%$ yield; ${ }^{1} \mathrm{H}-\mathrm{NMR}\left(\mathrm{CD}_{3} \mathrm{CN}, 600 \mathrm{MHz}\right) \delta 7.89$ $(\mathrm{d}, J=8.4 \mathrm{~Hz}, 2 \mathrm{H}), 7.72(\mathrm{~s}, 1 \mathrm{H}), 7.70-7.64(\mathrm{~m}, 3 \mathrm{H}), 7.59(\mathrm{dd}, J=8.0,0.9 \mathrm{~Hz}, 1 \mathrm{H}), 7.50(\mathrm{~d}, J=7.9 \mathrm{~Hz}, 1 \mathrm{H})$, $7.44(\mathrm{t}, J=7.9 \mathrm{~Hz}, 2 \mathrm{H}), 7.40(\mathrm{t}, J=7.9 \mathrm{~Hz}, 1 \mathrm{H}), 7.25(\mathrm{t}, J=7.4 \mathrm{~Hz}, 1 \mathrm{H}), 7.03(\mathrm{~d}, J=7.4 \mathrm{~Hz}, 2 \mathrm{H}), 5.68$ $(\mathrm{s}, 2 \mathrm{H}), 5.22(\mathrm{~s}, 2 \mathrm{H}) ;{ }^{13} \mathrm{C}-\mathrm{NMR}\left(\mathrm{CD}_{3} \mathrm{CN}, 151 \mathrm{MHz}\right) \delta 166.76,150.52,148.57,143.33,141.46,136.65,133.33$, 131.68, 130.26, 129.62, 129.39, 128.72, 127.01, 125.85, 124.11, 123.26, 121.70, 46.43; HRMS (ESI): Calcd. for $[\mathrm{M}+\mathrm{Na}]^{+} \mathrm{C}_{23} \mathrm{H}_{18} \mathrm{BrN}_{3} \mathrm{O}_{3} \mathrm{~S}_{2}$ : 526.9973, Found 526.9967.

4-(((Z)-4-oxo-2-(phenylimino)-5-((Z)-3-(trifluoromethyl)benzylidene)thiazolidin-3-yl)methyl)benzenesulfonami de (7): Light yellow powder, m.p. 226.6-227.4 ${ }^{\circ} \mathrm{C}, 44 \%$ yield; ${ }^{1} \mathrm{H}-\mathrm{NMR}\left(\mathrm{CD}_{3} \mathrm{CN}, 600 \mathrm{MHz}\right) \delta 7.89$ $(\mathrm{d}, J=8.4 \mathrm{~Hz}, 2 \mathrm{H}), 7.72(\mathrm{~s}, 1 \mathrm{H}), 7.66(\mathrm{~d}, J=8.4 \mathrm{~Hz}, 2 \mathrm{H}), 7.52(\mathrm{~s}, 1 \mathrm{H}), 7.47-7.41(\mathrm{~m}, 5 \mathrm{H}), 7.25(\mathrm{t}, J=7.5$ $\mathrm{Hz}, 1 \mathrm{H}), 7.02(\mathrm{dd}, J=8.3,0.9 \mathrm{~Hz}, 2 \mathrm{H}), 5.68(\mathrm{~s}, 2 \mathrm{H}), 5.22(\mathrm{~s}, 2 \mathrm{H}) ;{ }^{13} \mathrm{C}-\mathrm{NMR}\left(\mathrm{CD}_{3} \mathrm{CN}, 151 \mathrm{MHz}\right) \delta 165.23$, $149.00,147.03,141.78,139.92,134.86,133.65,129.94,128.92-128.62,128.15,127.84,126.84,125.47,124.30$, 122.59, 120.15, 44.89; HRMS (ESI): Calcd. for [M + Na] ${ }^{+} \mathrm{C}_{24} \mathrm{H}_{18} \mathrm{~F}_{3} \mathrm{~N}_{3} \mathrm{O}_{3} \mathrm{~S}_{2}$ : 517.0742, Found 517.0760.

4-(((Z)-5-((Z)-3-hydroxybenzylidene)-4-oxo-2-(phenylimino)thiazolidin-3-yl)methyl)benzenesulfonamide (8): Yellow powder, m.p. $268.2-269.3{ }^{\circ} \mathrm{C}, 30.30 \%$ yield; ${ }^{1} \mathrm{H}-\mathrm{NMR}$ (DMSO- $\left.d_{6}, 400 \mathrm{MHz}\right) \delta 9.83(\mathrm{~s}, 1 \mathrm{H}$ ), 7.83 $(\mathrm{d}, J=8.3 \mathrm{~Hz}, 2 \mathrm{H}), 7.70(\mathrm{~s}, 1 \mathrm{H}), 7.59(\mathrm{~d}, J=8.3 \mathrm{~Hz}, 2 \mathrm{H}), 7.48-7.24(\mathrm{~m}, 5 \mathrm{H}), 7.20(\mathrm{t}, J=7.4 \mathrm{~Hz}, 1 \mathrm{H})$, $7.00(\mathrm{t}, J=7.8 \mathrm{~Hz}, 3 \mathrm{H}), 6.92(\mathrm{t}, J=1.8 \mathrm{~Hz}, 1 \mathrm{H}), 6.84(\mathrm{dd}, J=8.1,1.7 \mathrm{~Hz}, 1 \mathrm{H}), 5.17(\mathrm{~s}, 2 \mathrm{H}) ;{ }^{13} \mathrm{C}-\mathrm{NMR}$ (DMSO- $\left.d_{6}, 101 \mathrm{MHz}\right) \delta 166.24,158.35,150.14,147.99,143.77,140.29,134.81,131.58,130.82,130.02$, $128.46,126.46,125.47,121.84,121.46,121.11,118.05,115.96,45.87$; HRMS (ESI): Calcd. for [M + Na] ${ }^{+}$ $\mathrm{C}_{23} \mathrm{H}_{19} \mathrm{~N}_{3} \mathrm{O}_{4} \mathrm{~S}_{2}: 465.0817$, Found 465.0814.

4-(((Z)-5-((Z)-4-fluorobenzylidene)-4-oxo-2-(phenylimino)thiazolidin-3-yl)methyl)benzenesulfonamide (9): Light yellow powder, m.p. $209.7-211.0{ }^{\circ} \mathrm{C}, 33 \%$ yield; ${ }^{1} \mathrm{H}-\mathrm{NMR}\left(\mathrm{CD}_{3} \mathrm{CN}, 600 \mathrm{MHz}\right) \delta 7.92-7.83$ $(\mathrm{m}, 2 \mathrm{H}), 7.77(\mathrm{~s}, 1 \mathrm{H}), 7.66(\mathrm{~d}, J=8.5 \mathrm{~Hz}, 2 \mathrm{H}), 7.60-7.52(\mathrm{~m}, 2 \mathrm{H}), 7.47-7.39(\mathrm{~m}, 2 \mathrm{H}), 7.27-7.18(\mathrm{~m}, 3 \mathrm{H})$, 7.07-6.98 (m, 2H), $5.69(\mathrm{~s}, 2 \mathrm{H}), 5.22(\mathrm{~s}, 2 \mathrm{H}) ;{ }^{13} \mathrm{C}-\mathrm{NMR}\left(\mathrm{CD}_{3} \mathrm{CN}, 151 \mathrm{MHz}\right) \delta 166.82,164.57,162.91$, 150.70, 148.54, 143.15, 141.42, 132.73, 130.75, 130.09, 129.20, 126.87, 125.62, 121.92, 121.60, 116.87, 116.72, 46.20; HRMS (ESI): Calcd. for $[\mathrm{M}+\mathrm{Na}]^{+} \mathrm{C}_{23} \mathrm{H}_{18} \mathrm{FN}_{3} \mathrm{O}_{3} \mathrm{~S}_{2}$ : 467.0774, Found 467.0771.

4-(((Z)-5-((Z)-4-hydroxybenzylidene)-4-oxo-2-(phenylimino)thiazolidin-3-yl)methyl)benzenesulfonamide (10): Yellow powder, m.p. $176.3-178.2{ }^{\circ} \mathrm{C}, 30 \%$ yield; ${ }^{1} \mathrm{H}-\mathrm{NMR}$ (DMSO- $\left.d_{6}, 400 \mathrm{MHz}\right) \delta 7.83(\mathrm{~d}, J=8.3 \mathrm{~Hz}, 2 \mathrm{H}$ ), $7.68(\mathrm{~s}, 1 \mathrm{H}), 7.57(\mathrm{~d}, J=8.3 \mathrm{~Hz}, 2 \mathrm{H}), 7.40(\mathrm{t}, J=7.8 \mathrm{~Hz}, 2 \mathrm{H}), 7.35(\mathrm{~d}, J=8.7 \mathrm{~Hz}, 2 \mathrm{H}), 7.19(\mathrm{t}, J=7.4 \mathrm{~Hz}, 1 \mathrm{H})$, $7.00(\mathrm{~d}, J=7.4 \mathrm{~Hz}, 2 \mathrm{H}), 6.84(\mathrm{~d}, J=8.7 \mathrm{~Hz}, 2 \mathrm{H}), 5.15(\mathrm{~s}, 2 \mathrm{H}) ;{ }^{13} \mathrm{C}-\mathrm{NMR}\left(\mathrm{DMSO}-d_{6}, 101 \mathrm{MHz}\right) \delta 166.52$, 162.30, 150.49, 148.20, 143.72, 140.50, 132.79, 132.24, 129.98, 128.37, 126.45, 125.27, 123.24, 121.51, 117.31, 115.24, 46.47; HRMS (ESI): Calcd. for $[\mathrm{M}+\mathrm{Na}]^{+} \mathrm{C}_{23} \mathrm{H}_{19} \mathrm{~N}_{3} \mathrm{O}_{4} \mathrm{~S}_{2}$ : 465.0817, Found 465.0824.

4-(((Z)-5-((Z)-4-methoxybenzylidene)-4-oxo-2-(phenylimino)thiazolidin-3-yl)methyl)benzenesulfonamide (11): Light yellow powder, m.p. $210.6-211.8{ }^{\circ} \mathrm{C}, 39 \%$ yield; ${ }^{1} \mathrm{H}-\mathrm{NMR}\left(\mathrm{CD}_{3} \mathrm{CN}, 600 \mathrm{MHz}\right) \delta 7.88$ $(\mathrm{d}, J=8.4 \mathrm{~Hz}, 2 \mathrm{H}), 7.74(\mathrm{~s}, 1 \mathrm{H}), 7.66(\mathrm{~d}, J=8.4 \mathrm{~Hz}, 2 \mathrm{H}), 7.43(\mathrm{t}, J=7.9 \mathrm{~Hz}, 2 \mathrm{H}), 7.23(\mathrm{t}, J=7.4 \mathrm{~Hz}, 1 \mathrm{H})$, 7.14-7.08 (m, 2H), $7.03(\mathrm{dd}, J=7.8,2.9 \mathrm{~Hz}, 3 \mathrm{H}), 5.68(\mathrm{~s}, 1 \mathrm{H}), 5.22(\mathrm{~s}, 2 \mathrm{H}), 3.85(\mathrm{~s}, 3 \mathrm{H}), 3.81(\mathrm{~s}, 3 \mathrm{H})$; ${ }^{13} \mathrm{C}-\mathrm{NMR}\left(\mathrm{CD}_{3} \mathrm{CN}, 151 \mathrm{MHz}\right) \delta$ 167.04, 161.70, 151.08, 148.70, 143.09, 141.57, 132.43, 131.25, 130.05, 
129.13, 126.85, 126.70, 125.50, 121.63, 119.00, 115.24, 55.79, 46.08; HRMS (ESI): Calcd. for [M + Na] ${ }^{+}$ $\mathrm{C}_{24} \mathrm{H}_{21} \mathrm{~N}_{3} \mathrm{O}_{4} \mathrm{~S}_{2}: 479.0973$, Found 479.0981.

4-(((Z)-5-((Z)-4-(benzyloxy)benzylidene)-4-oxo-2-(phenylimino)thiazolidin-3-yl)methyl)benzenesulfonamide (12): Light yellow powder, m.p. $219.6-221.3{ }^{\circ} \mathrm{C}, 48 \%$ yield; ${ }^{1} \mathrm{H}-\mathrm{NMR}\left(\mathrm{CD}_{3} \mathrm{CN}, 600 \mathrm{MHz}\right) \delta 7.88$ $(\mathrm{d}, J=8.4 \mathrm{~Hz}, 2 \mathrm{H}), 7.74(\mathrm{~s}, 1 \mathrm{H}), 7.66(\mathrm{~d}, J=8.4 \mathrm{~Hz}, 2 \mathrm{H}), 7.43(\mathrm{t}, J=7.9 \mathrm{~Hz}, 2 \mathrm{H}), 7.23(\mathrm{t}, J=7.4 \mathrm{~Hz}, 1 \mathrm{H})$, 7.14-7.08 (m, 2H), $7.03(\mathrm{dd}, J=7.8,2.9 \mathrm{~Hz}, 3 \mathrm{H}), 5.68(\mathrm{~s}, 1 \mathrm{H}), 5.22(\mathrm{~s}, 2 \mathrm{H}), 3.85(\mathrm{~s}, 3 \mathrm{H}), 3.81(\mathrm{~s}, 3 \mathrm{H})$; ${ }^{13} \mathrm{C}-\mathrm{NMR}\left(\mathrm{CD}_{3} \mathrm{CN}, 151 \mathrm{MHz}\right) \delta 167.00,151.56,151.00,149.88,148.60,143.09,141.59,131.54,130.04$, 129.13, 126.91, 125.51, 123.57, 121.65, 119.34, 114.33, 112.38, 57.51, 56.01, 46.09; HRMS (ESI): Calcd. for $[\mathrm{M}+\mathrm{Na}]^{+} \mathrm{C}_{30} \mathrm{H}_{25} \mathrm{~N}_{3} \mathrm{O}_{4} \mathrm{~S}_{2}:$ 555.1286, Found 555.1279.

4-(((Z)-5-((Z)-4-isopropylbenzylidene)-4-oxo-2-(phenylimino)thiazolidin-3-yl)methyl)benzenesulfonamide (13): Light yellow powder, m.p. 200.7-201.6 ${ }^{\circ} \mathrm{C}, 49 \%$ yield; ${ }^{1} \mathrm{H}$ NMR $\left(\mathrm{CD}_{3} \mathrm{CN}, 600 \mathrm{MHz}\right) \delta 7.92-7.84(\mathrm{~m}, 2 \mathrm{H})$, $7.76(\mathrm{~s}, 1 \mathrm{H}), 7.65(\mathrm{~d}, J=8.5 \mathrm{~Hz}, 2 \mathrm{H}), 7.48-7.40(\mathrm{~m}, 4 \mathrm{H}), 7.36(\mathrm{~d}, J=8.3 \mathrm{~Hz}, 2 \mathrm{H}), 7.26-7.20(\mathrm{~m}, 1 \mathrm{H})$, 7.06-6.98 (m, 2H), $5.68(\mathrm{~s}, 2 \mathrm{H}), 5.21(\mathrm{~s}, 2 \mathrm{H}), 2.95(\mathrm{hept}, J=6.9 \mathrm{~Hz}, 1 \mathrm{H}), 1.23(\mathrm{~d}, J=6.9 \mathrm{~Hz}, 6 \mathrm{H}) ;{ }^{13} \mathrm{C}-\mathrm{NMR}$ $\left(\mathrm{CD}_{3} \mathrm{CN}, 151 \mathrm{MHz}\right) \delta 166.94,152.13,150.99,148.60,143.11,141.49,131.83,131.38,130.63,130.05$, 129.17, 127.83, 126.85, 125.54, 121.61, 121.01, 46.11, 34.38, 23.46; HRMS (ESI): Calcd. for [M + Na] ${ }^{+}$ $\mathrm{C}_{26} \mathrm{H}_{25} \mathrm{~N}_{3} \mathrm{O}_{3} \mathrm{~S}_{2}:$ 491.1337, Found 493.1343.

4-(((Z)-5-((Z)-3,4-dimethoxybenzylidene)-4-oxo-2-(phenylimino)thiazolidin-3-yl)methyl)benzenesulfonamide (14): Light yellow powder, m.p. $249.8-250.9{ }^{\circ} \mathrm{C}, 41 \%$ yield; ${ }^{1} \mathrm{H}-\mathrm{NMR}\left(\mathrm{CD}_{3} \mathrm{CN}, 600 \mathrm{MHz}\right) \delta 7.88$ $(\mathrm{d}, J=8.4 \mathrm{~Hz}, 2 \mathrm{H}), 7.74(\mathrm{~s}, 1 \mathrm{H}), 7.66(\mathrm{~d}, J=8.4 \mathrm{~Hz}, 2 \mathrm{H}), 7.43(\mathrm{t}, J=7.9 \mathrm{~Hz}, 2 \mathrm{H}), 7.23(\mathrm{t}, J=7.4 \mathrm{~Hz}, 1 \mathrm{H})$, 7.14-7.08 (m, 2H), $7.03(\mathrm{dd}, J=7.8,2.9 \mathrm{~Hz}, 3 \mathrm{H}), 5.68(\mathrm{~s}, 1 \mathrm{H}), 5.22(\mathrm{~s}, 2 \mathrm{H}), 3.85(\mathrm{~s}, 3 \mathrm{H}), 3.81(\mathrm{~s}, 3 \mathrm{H})$; ${ }^{13} \mathrm{C}-\mathrm{NMR}\left(\mathrm{CD}_{3} \mathrm{CN}, 151 \mathrm{MHz}\right) \delta 167.00,151.56,151.00,149.88,148.60,143.09,141.59,131.54,130.04$, 129.13, 126.91, 125.51, 123.57, 121.65, 119.34, 114.33, 112.38, 57.51, 56.01, 46.09; HRMS (ESI): Calcd. for $[\mathrm{M}+\mathrm{Na}]^{+} \mathrm{C}_{25} \mathrm{H}_{23} \mathrm{~N}_{3} \mathrm{O}_{5} \mathrm{~S}_{2}$ : 509.1079, Found 509.1092.

\section{2. hCA Enzyme Inhibition Assays}

Carbonic anhydrases are able to catalyze the conversion 4-nitrophenyl acetate (4-NPA) to 4-nitrophenol. According to the method described previously by Verpoorte, the rate of this reaction is monitored spectrophotometrically, at $405 \mathrm{~nm}$, with a Perkin Elmer Envision 2104 plate reader [30,31] (Perkin Elmer, Waltham, MA, USA). 1X assay buffer consisted of $15 \mathrm{mmol} / \mathrm{L}$ 4-(2-hydroxyethyl)-1-piperazineethanesulfonic acid (HEPES) $(\mathrm{pH}=7.40) 0.01 \%$ tetraethylene glycol monododecyl ether (Brij) and $100 \mathrm{mmol} / \mathrm{L} \mathrm{NaCl}$. Recombinant human carbonic anhydrase II and IX was commercially available (Sino Biological Inc, Beijing, China) and prepared in 1X assay buffer with a concentration of $11.10 \mathrm{ng} / \mu \mathrm{L}$. Then, $18 \mu \mathrm{L}$ of enzyme solution was transferred into 384-well assay plates in triplicate. Stock solutions of the inhibitor $(10.00 \mathrm{mmol} / \mathrm{L})$ were prepared using DMSO as solvent, and then diluted 1:3 with DMSO. Ten different inhibitor concentrations were used: 600.00 $\mu \mathrm{mol} / \mathrm{L}, 200.00 \mu \mathrm{mol} / \mathrm{L}, 66.67 \mu \mathrm{mol} / \mathrm{L}, 22.22 \mu \mathrm{mol} / \mathrm{L}, 7.41 \mu \mathrm{mol} / \mathrm{L}, 2.47 \mu \mathrm{mol} / \mathrm{L}, 0.82 \mu \mathrm{mol} / \mathrm{L}, 0.27 \mu \mathrm{mol} / \mathrm{L}$, $0.091 \mu \mathrm{mol} / \mathrm{L}, 0.03 \mu \mathrm{mol} / \mathrm{L}$, and $0 \mu \mathrm{mol} / \mathrm{L}$. A volume of $2.00 \mu \mathrm{L}$ of each inhibitor was added into the assay solution. All compounds were allowed to incubate with the enzyme for $15 \mathrm{~min}$ at $25^{\circ} \mathrm{C}$ to form the Enzyme-Inhibitor (E-I) complex. After that, substrate 4-NPA $(1.00 \mathrm{mmol} / \mathrm{L}, 20.00 \mu \mathrm{L}$, Sigma-Aldrich, St. Louis, MI, USA) was added into the E-I complex solution and incubated for $90 \mathrm{~min}$ at $25^{\circ} \mathrm{C}$. The absorbance of each compound was measured with Envision 2104 plate reader. The inhibitor AZM and SLC-0111 were used as standards to investigate the inhibitory activity of these compounds.

\subsection{Single-Crystal Structure}

The configuration of the target compound was determined by the single-crystal X-ray diffraction (SuperNova, Rigaku, Tokyo, Japan) of compound (13). The compound crystallized in a centrosymmetric space groups as a triclinic system. The resulting structure is shown in Figure 5 (CCDC Number: 1888237). The experiment showed that the double bonds between N2-C10 and C9-C17 are both in the $\mathrm{Z}$ configuration. 
A

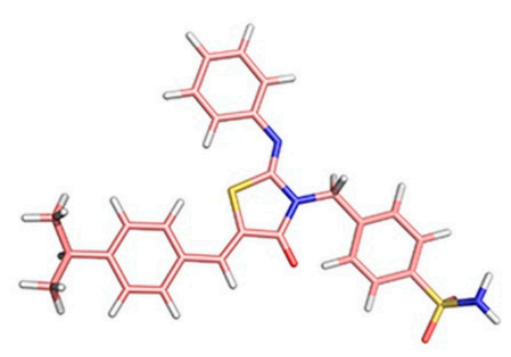

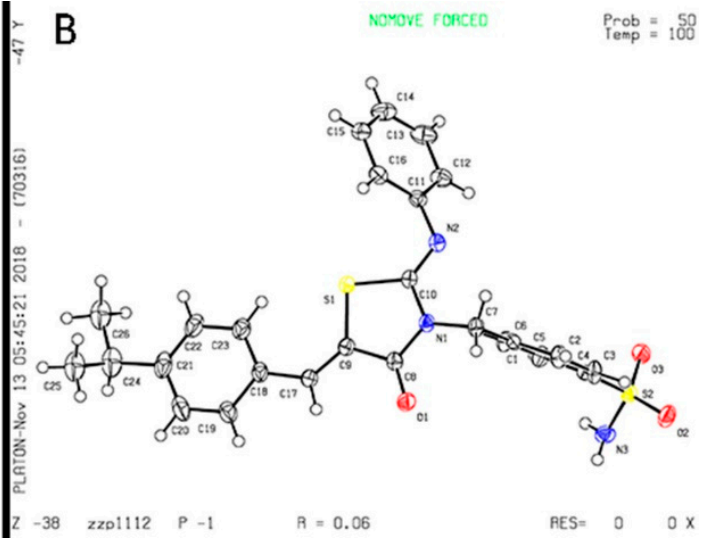

Figure 5. The molecular structure (A) and single-crystal structure (B) of compound (13).

\subsection{Preparation of Compound 2-14 for Docking Studies}

Molecular docking calculations using the ligand molecules with CA IX (PDB code: 5FL4) [32] and CA II (PDB: 4IWZ) [33] were conducted using the modified version of Autodock 4(Zn) [34,35]. A grid box with a grid spacing of $0.375 \AA$ was generated to define the binding pocket. AutoGrid 4 was used as the auxiliary program to generate affinity grid fields with the modified forcefield to generate maps files. Compound structures were built and minimized with the Accelrys Discovery Studio 3.0 software package [36] with flexible torsions assigned, and all dihedral angles were able to freely rotate. We applied the Lamarckian genetic algorithm to determine the appropriate binding positions, orientations, and conformations of the ligands. The optimized parameters were as follows: The maximum number of energy evaluations was increased to 25,000,000 per run, the iterations of Solis and Wets local search were 3000, the number of individuals in the population was 300 , and the number of generations was 25 . The results differing by $<2 \AA$ in a positional root mean square deviation were clustered together. In each group, the lowest binding energy configuration with the highest percentage frequency was selected as the group representative. All other parameters were maintained as default parameters.

\section{Conclusions}

A series of benzenesulfonamide-thiazolidinone derivatives was synthesized and evaluated for the ability to inhibit $h$ CA II and IX in vitro. Although the target compounds showed less selectivity between CA II and IX, the inhibitory activities of most derivatives were equivalent to AZM but were much higher than that of the precursor compound. The conclusion of SARs indicates that the introduction of heterocyclic rings in the molecules can increase CA inhibitory activity and is worthy of being further explored. In addition, the molecular docking results of $h$ CA II and IX indicated that the benzenesulfonamide derivatives containing thiazolidinone were structurally rigid and extended into the active sites of CAs with relatively fixed conformations. Such a rigid and relatively fixed conformation could interact with the key residue Val130 in the active site of CA IX, and without interacting with Phe130 in CA II. Further exploration around this point of view is expected to help improve the CA IX/II selectivity of this class of structure.

Supplementary Materials: The following are available online at http://www.mdpi.com/1420-3049/24/13/2418/s1, Table S1. Crystal data and structure refinement for compound 13; Figure S1-Figure S26. The ${ }^{1} \mathrm{H}-\mathrm{NMR}$ and ${ }^{13}$ C-NMR of compound 2-14.

Author Contributions: M.-S.C. and Y.L. supervised the whole experiment and provided technical guidance. Z.-P.Z. designed and synthesized all of novel compounds. Z.-F.Y., J.-Y.L., Z.-P.W. and Q.-J.W. assisted in the synthetic and biology research. J.W. supervised and carried out molecular modeling experiments.

Funding: We gratefully acknowledge the financial support from National Natural Science Foundation of China $(81473087,81872752)$. Dr. Yang Liu wishes to express his thanks for the support by the Program for 
Liaoning Innovative Talents in University (LR2017043) and the Career Development Support Plan for Young and Middle-aged Teachers in Shenyang Pharmaceutical University (ZQN2016005).

Conflicts of Interest: The authors declare no conflict of interest.

\section{References}

1. Stadie, W.C.; O'Brien, H. The catalytic of the hydration of carbon dioxide and dehydration of carbonic acid by an enzyme isolated from red blood cells. J. Biol. Chem. 1933, 103, 521-529.

2. Supuran, C.T. Structure and function of carbonic anhydrases. Biochem. J. 2016, 473, 2023-2032. [CrossRef] [PubMed]

3. Carbonic Anhydrase: Mechanism, Regulation, Links to Disease, and Industrial Applications, Subcellular Biochemistry; Frost, S.C.; McKenna, R., Eds.; Springer: Berlin/Heidelberg, Germany, 2014.

4. Švastová, E.; Hulíková, A.; Rafajová, M.; Zat'Ovičová, M.; Gibadulinová, A.; Casini, A.; Cecchi, A.; Scozzafava, A.; Supuran, C.T.; Pastorek, J.; et al. Hypoxia activates the capacity of tumor-associated carbonic anhydrase IX to acidify extracellular pH. FEBS Lett. 2004, 577, 439-445. [CrossRef] [PubMed]

5. Mboge, M.Y.; Mahon, B.P.; McKenna, R.; Frost, S.C. Carbonic Anhydrases: Role in pH Control and Cancer. Metabolites 2018, 8, 19.

6. Chiche, J.; Ilc, K.; Laferrière, J.; Trottier, E.; Dayan, F.; Mazure, N.M.; Brahimi-Horn, M.C.; Pouysségur, J. Hypoxia-Inducible Carbonic Anhydrase IX and XII Promote Tumor Cell Growth by Counteracting Acidosis through the Regulation of the Intracellular pH. Cancer Res. 2009, 69, 358-368. [CrossRef] [PubMed]

7. Swietach, P.; Vaughan-Jones, R.D.; Harris, A.L.; Hulikova, A.; Harris, A.L.; Harris, A. The chemistry, physiology and pathology of $\mathrm{pH}$ in cancer. Philos. Trans. R. Soc. B Boil. Sci. 2014, 369, 20130099. [CrossRef] [PubMed]

8. Neri, D.; Supuran, C.T. Interfering with $\mathrm{pH}$ regulation in tumours as a therapeutic strategy. Nat. Rev. Drug Discov. 2011, 10, 767-777. [CrossRef] [PubMed]

9. Supuran, C.T. Carbonic anhydrases. Bioorg. Med. Chem. 2013, 21, 1377-1378. [CrossRef] [PubMed]

10. Swietach, P.; Vaughan-Jones, R.D.; Harris, A.L. Regulation of tumor $\mathrm{pH}$ and the role of carbonic anhydrase 9. Cancer Metastasis Rev. 2007, 26, 299-310. [CrossRef] [PubMed]

11. Pastorekova, S.; Gillies, R.J. The role of carbonic anhydrase IX in cancer development: Links to hypoxia, acidosis, and beyond. Cancer Metastasis Rev. 2019, 1-13. [CrossRef]

12. A Phase I, Multi-center, Open-label, Study to Investigate the Safety, Tolerability and Pharmacokinetic of SLC-0111 in Subjects with Advanced Solid Tumours. 2016. Available online: https://clinicaltrials.gov/ct2/ show/NCT02215850 (accessed on 15 September 2017).

13. Supuran, C.T. Carbonic Anhydrase Inhibition and the Management of Hypoxic Tumors. Metabolites 2017, 7, 48. [CrossRef] [PubMed]

14. Andreucci, E.; Ruzzolini, J.; Peppicelli, S.; Bianchini, F.; Laurenzana, A.; Carta, F.; Supuran, C.T.; Calorini, L. The carbonic anhydrase IX inhibitor SLC-0111 sensitises cancer cells toconventional chemotherapy. J. Enzym. Inhib. Med. Chem. 2019, 8, 117-123.

15. Romagnoli, R.; Baraldi, P.G.; Prencipe, F.; Oliva, P.; Baraldi, S.; Salvador, M.K.; Lopez-Carz, L.C.; Brancale, A.; Ferla, S.; Hamel, E.; et al. Synthesis and biological evaluation of 2-methyl-4, 5-disubstituted oxazoles as a novel class of highly potent antitubulin agents. Sci. Rep. 2017, 7, 1-19. Available online: https://www.nature.com/articles/srep46356. (accessed on 13 April 2017). [CrossRef] [PubMed]

16. Li, Y.-S.; Hu, D.-K.; Zhao, D.-S.; Liu, X.-Y.; Jin, H.-W.; Song, G.-P.; Cui, Z.-N.; Zhang, L.-H. Design, synthesis and biological evaluation of 2,4-disubstituted oxazole derivatives as potential PDE4 inhibitors. Bioorg. Med. Chem. 2017, 25, 1852-1859. [CrossRef] [PubMed]

17. Jin, R.; Sun, X.; Liu, Y.; Long, W.; Chen, B.; Shen, S.; Ma, H. Synthesis, crystal structure, biological activity and theoretical calculations of novel isoxazole derivatives. Spectrochim. Acta Part A Mol. Biomol. Spectrosc. 2016, 152, 226-232. [CrossRef] [PubMed]

18. Tomi, I.H.; Tomma, J.H.; Al-Daraji, A.H.; Al-Dujaili, A.H. Synthesis, characterization and comparative study the microbial activity of some heterocyclic compounds containing oxazole and benzothiazole moieties. J. Saudi Chem. Soc. 2015, 19, 392-398. [CrossRef]

19. Rouf, A.; Tanyeli, C. Bioactive thiazole and benzothiazole derivatives. Eur. J. Med. Chem. 2015, 97, 911-927. [CrossRef] [PubMed] 
20. Nakamura, T.; Sato, M.; Kakinuma, H.; Miyata, N.; Taniguchi, K.; Bando, K.; Koda, A.; Kameo, K. Pyrazole and Isoxazole Derivatives as New, Potent, and Selective 20-Hydroxy-5,8,11,14-eicosatetraenoic Acid Synthase Inhibitors. J. Med. Chem. 2003, 46, 5416-5427. [CrossRef]

21. Patel, N.B.; Shaikh, F.M. Synthesis and antimicrobial activity of new 4-thiazolidinone derivatives containing 2-amino-6-methoxybenzothiazole. Saudi Pharm. J. 2010, 18, 129-136. [CrossRef]

22. Shiradkar, M.R.; Ghodake, M.; Bothara, K.G.; Bhandari, S.V.; Nikalje, A.; Akula, K.C.; Desai, N.C.; Burange, P.J. Synthesis and anticonvulsant activity of clubbed thiazolidinoneebarbituric acid and thiazolidinoneetriazole derivatives. Arkivoc 2007, 14, 58-74.

23. Ottana, R.; Maccari, R.; Giglio, M.; Del Corso, A.; Cappiello, M.; Mura, U.; Cosconati, S.; Marinelli, L.; Novellino, E.; Sartini, S.; et al. Identification of 5-arylidene-4-thiazolidinone derivatives endowed with dual activity as aldose reductase inhibitors and antioxidant agents for the treatment of diabetic complications. Eur. J. Med. Chem. 2011, 46, 2797-2806. [CrossRef] [PubMed]

24. Jain, A.K.; Vaidya, A.; Ravichandran, V.; Kashaw, S.K.; Agrawal, R.K. Recent developments and biological activities of thiazolidinone derivatives: A review. Bioorg. Med. Chem. 2012, 20, 3378-3395.

25. Barreca, M.L.; Balzarini, J.; Chimirri, A.; De Clercq, E.; De Luca, L.; Höltje, H.D.; Höltje, M.; Monforte, A.M.; Monforte, P.; Pannecouque, C.; et al. Design, Synthesis, Structure-Activity Relationships, and Molecular Modeling Studies of 2,3-Diaryl-1,3-thiazolidin-4-ones as Potent Anti-HIV Agents. J. Med. Chem. 2002, 45, 5410-5413. [CrossRef] [PubMed]

26. Takasu, K.; Pudhom, K.; Kaiser, M.; Brun, R.; Ihara, M. Synthesis and Antimalarial Efficacy of Aza-Fused Rhodacyanines in Vitro and in the P. berghei Mouse Model. J. Med. Chem. 2006, 49, 4795-4798. [CrossRef] [PubMed]

27. Unsal-Tan, O.; Ozadali, K.; Piskin, K.; Balkan, A. Molecular modeling, synthesis and screening of some new 4-thiazolidinone derivatives with promising selective COX-2 inhibitory activity. Eur. J. Med. Chem. 2012, 57, 59-64. [CrossRef]

28. Ansari, M.F.; Idrees, D.; Hassan, M.I.; Ahmad, K.; Avecilla, F.; Azam, A. Design, synthesis and biological evaluation of novel pyridine-thiazolidinone derivatives as anticancer agents: Targeting human carbonic anhydrase IX. Eur. J. Med. Chem. 2018, 144, 544-556.

29. Güzel-Akdemir, Ö.; Angeli, A.; Demir, K.; Supuran, C.T.; Akdemir, A. Novel thiazolidinone-containing compounds, without the well-known sulphonamide zinc-binding group acting as human carbonic anhydrase IX inhibitors. J. Enzym. Inhib. Med. Chem. 2018, 33, 1299-1308.

30. Li, F.-R.; Fan, Z.-F.; Qi, S.-J.; Wang, Y.-S.; Wang, J.; Liu, Y.; Cheng, M.-S. Design, Synthesis, Molecular Docking Analysis, and Carbonic Anhydrase IX Inhibitory Evaluations of Novel N-Substituted- $\beta$-d-Glucosamine Derivatives that Incorporate Benzenesulfonamides. Molecules 2017, 22, 785.

31. Verpoorte, J.A.; Mehta, S.; Edsall, J.T. Esterase activities of human carbonic anhydrases B and C. J. Boil. Chem. 1967, 242, 4221-4229.

32. Leitans, J.; Kazaks, A.; Balode, A.; Ivanova, J.; Zalubovskis, R.; Supuran, C.T.; Tars, K. Efficient Expression and Crystallization System of Cancer-Associated Carbonic Anhydrase Isoform IX. J. Med. Chem. 2015, 58, 9004-9009. [CrossRef]

33. Biswas, S.; McKenna, R.; Supuran, C.T. Effect of incorporating a thiophene tail in the scaffold of acetazolamide on the inhibition of human carbonic anhydraseisoforms I, II, IX and XII. Bioorg. Med. Chem. Lett. 2013, 23, 5646-5649. [CrossRef] [PubMed]

34. Morris, G.M.; Huey, R.; Lindstrom, W.; Sanner, M.F.; Belew, R.K.; Goodsell, D.S.; Olson, A.J. AutoDock4 and AutoDockTools4: Automated Docking with Selective Receptor Flexibility. J. Comput. Chem. 2009, 30, 2785-2791. [CrossRef] [PubMed]

35. Santos-Martins, D.; Forli, S.; Ramos, M.J.; Olson, A.J. AutoDock4Zn: An Improved AutoDock Force Field for Small-Molecule Docking to Zinc Metalloproteins. J. Chem. Inf. Model. 2014, 54, 2371-2379.

36. Discovery Studio User Manual; Accelrys Inc.: San Diego, CA, USA, 2008.

Sample Availability: Samples of the compounds (2)-(14) are available from the authors. 